\title{
Comunicación digital en desarrollo turístico de la Isla Puná, Ecuador
}

\author{
Digital communication as a tourism development \\ of Puná Island, Ecuador
}

\author{
Karen Andrade Mendoza \\ Universidad Central del Ecuador \\ kiandrade@uce.edu.ec \\ https://orcid.org/0000-0002-9513-1614
}

\begin{abstract}
Resumen
Este estudio procura exponer la importancia de la comunicación digital e intercultural para la conservación de los recursos naturales así como para la preservación del patrimonio cultural, también priorizar la comunicación para potencializar actividades de desarrollo económico y social como el turismo, esto, a través del reconocimiento y visualización de las comunidades, por parte de un público más amplio, con el uso de las diversas herramientas comunicativas como las que proveen el internet y los espacios mediáticos.

Además, se caracterizará la riqueza natural y cultural existente en la Isla Puná, Ecuador, como artífice para un proceso de difusión de información y de ampliación de los canales de comunicación, entre los diversos sectores productivos, sociales y culturales presentes en las dinámicas poblacionales de los ciudadanos que la habitan.

La investigación se realizó a través de un análisis cualitativo de la información, con la aplicación de entrevistas y levantamiento de información etnográfica, en continuas visitas de campo, a fin de conocer de primera mano las riquezas de patrimonio natural y cultural existentes en la isla. Cabe acotar que, el estudio mostró debilidades por parte de las organizaciones en el uso las herramientas de comunicación digital, pero a la vez se identificó fuertes potencialidades en su aplicación para la difusión turística y conservación de las riquezas de la isla.
\end{abstract}

\section{Palabras clave}

Comunicación digital, turismo, conservación, patrimonio natural, patrimonio cultural, recursos naturales.

Forma sugerida de citar: Andrade Mendoza, Karen (2019). Comunicación digital en desarrollo turístico de la Isla Puná, Ecuador. Universitas, 30, pp. 211-227. 


\begin{abstract}
This study aims to expose the importance of digital and intercultural communication both for natural resources conservation and cultural heritage preservation, as well as communication is a priority to potentiate economic and social development activities such as; tourism, through recognition and visualization of communities by a wider audience, with the use of the various communication tools provided by the internet and media spaces.

In addition, the natural and cultural wealth existing on Puná Island is characterized as the architect through a process of information's dissemination and expansion of all the communication channels between the various productive, social and cultural sectors present in the population dynamics of its citizens, they inhabit it.

The research was carried out through a qualitative analysis of the information, with the application of interviews and the collection of ethnographic information, with continuous visits to the field in order to know first-hand the natural and cultural heritage riches existing on the island. It should be noted that the study showed weaknesses on the part of organizations in the digital communication tools, but at the same time, strong potentialities were identified with respect to its application for tourist dissemination and conservation of the island's riches.
\end{abstract}

Keywords

Digital communication, tourism, conservation, natural heritage, cultural heritage, natural resources.

\title{
Introducción
}

La comunicación es parte integral de la vida de los seres humanos. La mayor parte de nosotros disfrutamos de conocer a otras personas, otros lugares, paisajes, otras culturas. En el mundo actual, a través de la comunicación digital (con el uso del internet); la geografía, el espacio y el tiempo se han acortado. Ahora podemos estar comunicados a cualquier hora y desde cualquier lugar, donde la tecnología nos permita conectarnos en línea. El ejercicio de la comunicación implica formas de comportamiento e interacción social, tanto como individuos o como sociedad. Los humanos entablamos conocimiento uno con otros, así como de los espacios que habitamos, por esto la movilidad humana condujo al descubrimiento de nuevos territorios y experiencias (Labate \& Arrueta, 2017).

Este contacto nos brinda la posibilidad de conocer a otros, compartir nuestra cosmovisión y nuestro pasado, a más de intercambiar significaciones e información referente a nuestra historia y formas de vida. El viaje se transforma en un 
proceso de aprendizaje y educación, a la vez que, el turismo genera los recursos necesarios para mejorar las condiciones económicas y sociales de las poblaciones que ofertan servicios a más de la riqueza patrimonial natural y cultural.

La potencialización del turismo a través de la recuperación y exhibición del patrimonio cultural, se presenta como una alternativa viable para el impulso de la economía de las comunidades y de su fortalecimiento cultural y social. La conservación y la protección de los recursos naturales renovables y la transmisión de conocimientos culturales es importante para sostener nuestras sociedades y su conexión con nuestra ancestralidad. Los habitantes de la localidad, tienen necesidad y urgencia en compartir sus historias y recobrar su memoria, con todo su grupo social y con el resto de la sociedad.

El compartir su cultura es un aspecto relevante para los pueblos, esto se conjuga con la comunicación, a los seres humanos nos motivan lo visual y lo auditivo. "Con la aparición de nuevos actores, la acelerada expansión de las redes sociales, la explosión de los macrodatos (big data) y de la computación en nube, han surgido nuevos modelos de creación, producción, distribución, acceso y participación” (Kulesz, 2017, p. 5). Por esta razón, muchos pueblos buscan ser visualizados en los medios de comunicación (incluido las redes sociales) y de esa forma impulsar el conocimiento y la curiosidad respecto de su patrimonio y tradiciones.

Muchas comunidades y poblaciones buscan potencializar su desarrollo económico y social con la incursión en el mundo turístico, donde la promoción por redes sociales y otros medios del internet se transforma en un elemento transcendental en la actualidad, especialmente a la hora de publicitar su patrimonio cultural y natural sin depender de empresas o agencias turística. Este es uno de los aspectos positivos del internet, ya que brinda autonomía respecto del gran capital e posibilita la incorporación de la diferencia (Moreno, 2015).

Hoy en día es muy complicado para los pueblos y sus sociedades preservar y proteger sus recursos, ya que, las poblaciones y sus autoridades se ven impulsadas a incorporar actividades extractivas, las que en su gran mayoría son perjudiciales para el ambiente y sus distintos hábitats, tal como es la industria camaronera y la actividad pesquera. Esto conlleva una urgencia respecto a la conservación del patrimonio y la búsqueda de nuevas alternativas económicas, con el impulso del turismo se potencializa la incorporación de gran parte de la población local a dinámicas capitalistas, al permitir la observación y comercialización de sus recursos naturales y culturales, a través de la promoción de ser punto de visita para muchos turistas, como objetivo de sus viajes. 
La posibilidad de conocer otras culturas y de establecer comunicación con otras personas, es uno de los aspectos más relevantes en el ejercicio de un viaje o del realizar turismo. En el mundo actual gran cantidad de personas desean viajar a lugares fantásticos llenos de naturaleza y expresiones culturales, pero es claro que, los efectos del turismo tienen implicaciones tanto en las sociedades como en las decisiones de gobierno sobre los territorios y recursos existentes en los mismos.

El turismo se avizora como una salida a los problemas de subsistencia, pero el desarrollo de esa capacidad se dificulta ante las necesidades prioritarias de una población, y el impulso de actividades productivas que generan impactos adversos en la conservación del ambiente. Este estudio procura exponer la importancia de la comunicación digital para la promoción del área, como también enfocar a la comunicación como parte prioritaria para potencializar actividades de desarrollo como el turismo, bajo la consideración que tanto la exposición digital y su incursión en dicha actividad implica afrontar impactos sociales, culturales y ambientales para la población, es decir la tecnología se transforma en un aspecto importante para el impulso de la economía social (Deux \& Vannini, 2016, p. 38).

Con la finalidad de mostrar las complejidades implícitas en el problema planteado en el párrafo anterior, se presentará el caso de la Isla Puná en el golfo de Guayaquil. Se plasma el estado situacional actual de sus pobladores y comprometer posibles acciones favorables al desarrollo de las poblaciones de la parroquia Puná, esto bajo la sombrilla de la mediatización de la comunicación. Este estudio se sustenta en el levantamiento de información de primera mano, a través de visitas y contacto directo con los dirigentes, a más del apoyo de la revisión bibliográfica referente a todos los temas subyacentes a la discusión, debe aclararse que la información bibliográfica referente a la Isla y sus pobladores es escasa, lo que conllevo visitas constantes al sitio. Los datos presentados se analizan a partir del ámbito de la comunicación, la cultura y las actividades económicas ligadas al desarrollo de los pueblos, bajo un ejercicio etnográfico y teórico analítico.

\section{La comunicación el día de hoy y su relevancia para la transmisión cultural y desarrollo de las poblaciones}

La comunicación trasciende todos los espacios y se transforma en una herramienta al servicio de los pueblos, ya que, no sólo nos permite conocer- 
nos unos a otros sino también mostrar nuestras singularidades al público, lo que permite potencializar actividades como el turismo y la conservación del patrimonio natural e inmaterial. Todo aquel que busque visualizarse deberá prepararse para incursionar en nuevos universos y pensamientos, mostrando sus diversas facetas y propuesta. (Martínez, 2006). Las comunidades y grupos sociales se encuentran inmersos en esta dinámica, tanto el trabajo como el ocio están atados actualmente a la tecnología, aunque fuere solo para realizar una consulta.

La comunicación mediatiza y difunde la cultura, y esta última se alimenta también de la de cultura con la producción y consumo de signos, donde el ámbito de lo audiovisual contribuye en la difusión de información y recreación de la misma, lo que permite al espectador tener una primera experiencia frente a un nuevo escenario. esta forma, los pueblos y sociedades pueden servirse de los distintos medios de comunicación para compartir sus paisajes y promover actividades en sus comunidades, especialmente aquellas relacionadas al turismo y la conservación de los ecosistemas (Labate \& Arrueta, 2017; Martínez, 2006). El turismo contribuye totalmente a esta dinámica, permitiéndonos viajar dentro de la cosmovisión y entorno de otros pueblos.

El turismo reproduce la dominación de una cultura sobre otra. Los gustos y las preferencias reflejados en la oferta de estilos de viaje y estancias turísticas responden ante un tipo de demanda que tiende a estandarizar la oferta (Catalano \& Tottino, 2016). Este tipo de influencia y de modelo pone en peligro las expresiones culturales propias de las raíces de los isleños, en el caso particular de la parroquia Puná, situación que se conecta directamente con los procesos de globalización y capitalización de las relaciones sociales.

La cultura y su reproducción está ligada a los espacios de poder, donde el control de lo mediático define el acceso a proyectos o propuestas de desarrollo acordes al entorno y a las características sociales de la población, ya que, los productos son sometidos a una valoración desde sectores antagónicos en el espectro de lo social, económico y político. La cultura es un aspecto trascendental de las sociedades, ésta define la forma en cómo se comunican los grupos humanos y la información que se transmite hacia otros. Todas las expresiones humanas son producto de la cultura y son generadoras de rasgos y formas culturales (Harris (2007 [1989], p.17).

Para Manuel Castells (Torres, 2014, p. 355), una de las formas más eficientes de comunicación móvil es el internet con su universalización, ya que, es el medio más extensivo para la transmisión de las expresiones cultu- 
rales y fortalece la interactividad a través de lo digital, potencializando una globalización financiera y tecnológica, pero que impone relaciones de poder en el desarrollo de redes de comunicación interactiva.

En el mundo actual, los avances tecnológicos son una herramienta potencializadora para las economías populares y el desarrollo de actividades sociales, culturales, económicas y políticas. La información tiene la cualidad de estar estructurada por códigos culturales de referencia, y sus protocolos están interconectados con el proceso mismo de la comunicación (Kulesz, 2017; Labate \& Arrueta, 2017; Martínez, 2006). El internet se transforma en un aliado del turismo y de la conservación patrimonial, faculta a los pueblos a difundir información sobre su cultura e identidad, mostrándose al mundo y posicionándose en las sociedades actuales. Esta herramienta ofrece romper con el aislamiento tradicional de comunidades, posibilitando su apertura al mundo, y permite una comunicación fluida con otros actores y otras realidades, elimina barreras de espacio y tiempo, identidad y nivel socio-económico.

El conocer nuestra historia, a nuestros pueblos, nuestro territorio, se posiciona como uno de los aspectos más relevantes en el siglo que transcurre. La necesidad de compartir lo que somos y de reconocernos en nuestra propia cosmovisión e identidad, discurre en una multiplicidad de acciones que a la vez están encaminadas a mejorar la vida de las personas (Quiñones, 2005). La población de la Isla Puná y sus autoridades parroquiales impulsan acciones para ser conocidos y reconocidos por otros sectores de la población nacional e internacional, estas acciones en la actualidad deben estar conectadas al desarrollo de propuestas digitales que puedan difundirse por medio de las redes sociales, de tal manera que, toda la información respecto a la riqueza cultural y natural del territorio y sus habitantes llegue a conocimiento de un público más extenso a través del internet (Labate \& Arrueta, 2017; Sandoval, 2007).

Más las acciones de promoción local deben estar conectadas con la conservación de los recursos. El interés por conservar y mostrar el patrimonio que poseen los pueblos está presente en cada una de las comunidades que componen la parroquia rural Isla Puná. Esto implica no sólo incorporar herramientas comunicacionales que potencien la actividad turística, sino también llevar a cabo una labor constante de concientización de la población referente a su trato con el turista. Situación que resalta la importancia de que la sociedad puneña debe conocer su historia con sus referentes ancestrales, así como la riqueza natural y cultural de la cual son poseedores. 
Martín-Barbero, nos recuerda a Zigmunt Bauman y su idea sobre el turista, "el turista habita un mundo desespacializado, sin territorio, de ahí que su movilidad sea instantánea casi tanto como el tiempo en que vive, ese mundo en el que quedarse quieto es morir, y vivir es transitar incesantemente acumulando "nuevas" experiencias, sensaciones y emociones, el mundo del turista es en definitiva el del consumidor" (2015, p. 25). El turista incentiva la actividad económica, a la vez que pone en el mapa a las poblaciones con referente a la opinión que se emite sobre el sitio y su gente. El turismo se define como "el conjunto de actividades que realizan las personas a lo largo de un viaje que incluye un desplazamiento desde su lugar de residencia habitual hacia un lugar de destino" y sostiene un discurso hegemónico respecto a los beneficios del turismo y la forma en cómo se lo debe realizar (Catalano \& Tottino, 2016).

El éxito de una estrategia para incrementar las visitas a la Isla Puná es potencializar su imagen como una zona de gran diversidad, su cultura e historia e impulsar turísticamente sus recursos. En la actualidad, el turismo y los viajeros son un fenómeno de la globalización y del avance en los medios de comunicación. Especialmente, el internet y las redes sociales incrementaron la difusión de lugares y expandió la comprensión del espacio-tiempo, esto potencializó la movilidad humana y el flujo turístico (Cioce \& Silva, 2015; Díaz \& López, 2012).

Debe tenerse presente que, el turismo reproduce relaciones de poder y desigualdad. A pesar de que promete mejorar la vida de las poblaciones que brindan servicios, puede ser un instrumento de doble filo, pues tiene efectos sobre las poblaciones que puede conducir a procesos de aculturación y transculturación, como también a la afectación del medio ambiente. "La llamada industria del turismo ciertamente de fácil correlación con el proyecto civilizatorio industrial-tecnológico, sobrepone la preocupación económica a las de cuño socioambiental" (Cioce \& Silva, 2015, p. 7). Reconocer estos aspectos, brindan a las comunidades opciones de cómo enfrentarse y aprovechar la actividad turística, más esto no constituye un seguro que proteja la ancestralidad de los pueblos y su constructo histórico identitario. En el caso de la Puná, hasta el momento no hay inversiones de grandes capitales en el desarrollo turístico, a pesar de que las autoridades mantienen negociaciones con varias agencias de viajes no han logrado avances al respecto.

Uno de los aspectos en que incide la visualización de las culturas, es la comunicación y el uso de los medios necesarios para lograr la difusión de 
información. "La comunicación con otros pueblos, con otras geografías, con otras culturas se vuelve una mercancía, abriendo paso al turismo como una de las industrias más prósperas, dado que moviliza millones de personas" (Catalano \& Tottino, 2016). Esta actividad se presenta como movilizadora y generadora de capital, a la vez que, el turista cumple la función de captador y difusor de la diversidad cultural que encuentra en los sitios (Requena \& Muñoz, 2006). Esta experiencia puede ser encapsulada en el típico turismo tradicional, es importante brindar opciones y actividades que muestren las sociedades que les acogen. En el caso de la Isla Puná, las ofertas de actividades turísticas son amplias, abarcan desde actividades ecoturísticas, como es el turismo comunitario, así como también arqueológico y cultural.

En la siguiente sección se caracterizará la riqueza natural y cultural existente en la Isla Puná, como artífice para un proceso de difusión de información y de ampliación de los canales de comunicación entre los diversos sectores productivos, sociales y culturales presentes en las dinámicas poblacionales de los ciudadanos que la habitan.

\section{Caracterización de la situación de las poblaciones de la Isla Puná y su potencial turístico}

La Isla Puná pertenece a la parroquia La Puná y al cantón Guayaquil de la provincia del Guayas. La isla tiene una gran diversidad biológica, desde la presencia de planicies, bosques secos, manglares y playas. Una amplia extensión de camaroneras en el norte y el sureste de la isla, en conjunto con áreas agrícolas representan cerca de un $25 \%$ del territorio intervenido. En este territorio encontramos dos elevaciones importantes: el cerro Yanzún, al norte, y el cerro Zambapala, al sur. Los manglares que comprenden el 12,4\% de la superficie. En total, las áreas intervenidas por la población ocupan la cuarta parte de la Isla (Zabala, Nato \& Rosero, 2014). Casi la mitad de la isla está cubierta por dos tipos de bosque: Monte Espinoso Tropical se extiende desde el nivel del mar hasta los 300 msnm y la otra corresponde a Bosque Muy Seco Tropical que también se localiza entre las cotas 0 y 300 msnm, y ubicados principalmente en el centro de la isla (Chancay, 2017).

La superficie de la isla es de $920 \mathrm{~km}^{2}$, y cuenta con 22 comunidades entre las que sobresalen, Campo Alegre, Cauchiche, Puná Vieja, Puná Nueva, Agua Piedra, Estero de Boca, Bellavista, Río Hondo, etc. (Chancay, 2017, pp. 45-46). 
La parroquia cuenta con 7455 habitantes al 2015 y el territorio está ubicado en la unidad de síntesis territorial Corredor de bosque seco tropical Bahía- Manta-Salinas Puná. La estructura vial terrestre es básica. Se encuentran caminos de segundo y tercer orden que recorren algunos sectores de la isla, estos son caminos de verano ya que al invierno se hacen intransitables. Las vías de mayor uso carrozable son las playas de la isla. La sociedad isleña se encuentra altamente organizada, allí se identificaron 50 representantes de asociaciones que abarcan: comunas, cooperativas pesqueras, recintos, puertos, barrios, cangrejeros, agricultores, transporte y pro mejoras (PDOT Puná, 2015).

En la isla se realizan varias actividades económicas y productivas, ligadas a los ecosistemas circundantes. Por ejemplo, en las comunas de Campo Alegre, Agua Piedra, Bellavista y Cauchiche predominan los ecosistemas de manglar y costeros. En estas zonas se han identificado una significativa cantidad de especies arbóreas como: el laurel de Puná, el muyuyo, el roble, el algarrobo y el ceibo (Crespo, 2014).

Entre las actividades productivas relevantes encontramos a la agricultura. Uno de los cultivos más significativos de la isla es la chirimoya. Un poco menos de 200 familias poseen un aproximado de 200 hectáreas en la zona cercana a la cabecera parroquial. Al igual que, en varios de los otros sectores económicos, este grupo se encuentra organizado alrededor de la Asociación de Agricultores 23 de septiembre. Estos cultivos al igual que otros, como por ejemplo el maíz o la pitahaya, han sufrido deterioro constante en su rendimiento productivo a causa de la escases de lluvias en los últimos años. Las personas que disponen de agua dulce en los hogares, ésta proveniente de pozos, y quienes no los poseen deben comprarla a proveedores particulares, ya que, el agua es necesaria para el consumo humano, y la entubada es salobre.

La situación climática afecta también la ganadería de la zona, la ausencia de lluvias pone en jaque la sostenibilidad de las poblaciones. Esta gran isla sufre por sequías constantes que afectan la capacidad de desarrollo económico de la población y la misma conservación de los ecosistemas. Los cultivos dependen sólo de las lluvias y el acceso a agua dulce es escaso.

La actividad pesquera es muy importante en la zona, esto tiene un impacto directo sobre la biodiversidad marina de la zona, ya que la frecuencia de uso del recurso es continua e intensiva. Así también en la isla, existe una gran extensión de camaroneras, ubicadas principalmente en las líneas costeras del norte y sur, colindan con el área centro-este. Estas camaroneras 
se han transformado en un eje de conflicto dentro del área, pues varias comunas mantienen demandas por ocupación de sus territorios por la empresa privada y, la deforestación del manglar es continua como efecto de esta actividad económica.

La oferta de empleos es pequeña y la capacidad de endeudamiento de la mayor parte de la población es reducida. Incorporar nuevas ideas e iniciativas constituye una de las prioridades en la zona, es clara la urgencia de créditos, tanto para mejorar sus plantaciones como para invertir en negocios o servicios turísticos que permitan atraer visitantes. Pero esto se dificulta, ya que la comunicación entre las poblaciones es complicada, debido a la reducida cantidad de vías terrestres que conectan los distintos poblados y el estado deplorable de las mismas.

Otro de los aspectos relevantes es el patrimonio cultural de las poblaciones, el mismo que no sólo comprende el bagaje étnico e identitario de su población, sino también una gran cantidad de exploraciones e investigaciones arqueológicas realizadas en el territorio puneño. Los registros arqueológicos del área de la Isla Puná, se remonta al periodo de Integración, entre el 900 y 1500 d.C., lo que da registro de sociedades prehispánicas de larga trayectoria ligados a la Cultura Huancavilca (Guancavilca) (Delgado, 2014). Este patrimonio constituye un baluarte para la población, pues se podría potencializar museos de sitio que convocaran la visita de turistas a la zona.

Desde esa época prehispánica, los isleños explotan los recursos costeros y del área de manglar en actividades como la pesca, la recolección de crustáceos y moluscos, los recursos forestales y la agricultura. Varios estudios apuntan una alta densidad poblacional en la isla, aún en épocas tempranas, lo que implicaría transformaciones en los hábitats y en la calidad de vida de las poblaciones.

Estos asentamientos se produjeron paulatinamente desde los manglares de las orillas hacia las zonas boscosas internas, especialmente del área de los Cerros Yanzún y Zambapala, ubicados en el centro y Sur de la isla. Durante el contacto, los españoles relataron la presencia de una sociedad cacical que combinaba la producción de bienes locales, la extracción de recursos naturales y la elaboración de fina orfebrería (Volland, 1995, en Chancay, 2017, p. 26).

Dado este panorama parcial sobre las ocupaciones humanas, se encuentra un registro arqueológico sólido de "77 sitios, de los cuales, en relación a su filiación cultural, 2 son de una sola ocupación Valdivia, 2 son multicom- 
ponentes Valdivia-Jambelí, 1 de ocupación Chorrera, 2 multicomponentes Chorrera-Jambelí, 37 Jambelí y 33 Guancavilca" (Chancay, 2017, p. 27). Descripciones sobre el flujo y permanencia de los asentamientos a través de diversos siglos y momentos prehispánicos, son descritos durante el Periodo de Desarrollo Regional (Aleto \& Elwell, 1990, Sánchez, 2013) y en el periodo de la Integración en los sitios Yanzún y Cerro Zambapala (Álvarez, 2014). En estos sitios el registro constante es la cerámica, áreas ceremoniales y evidencia de viviendas.

El Instituto Nacional de Patrimonio Cultural- INPC Regional 5 ha desarrollado varios estudios e investigaciones en la Isla Puná. Entre ellos resalta en el 2010, el Proyecto Investigación Arqueológica en Campo Alegre, Isla Puná, Guayas, Ecuador.

El objetivo de este trabajo fue contextualizar la piedra tallada zoomorfa que actualmente, se encuentra en el Museo Municipal de la Ciudad de Guayaquil. Esta escultura fue sacada de su contexto original a inicios del siglo XX, localizado a $1.5 \mathrm{~km}$ de la Comuna de Campo Alegre (Chancay, 2017, p. 65).

En distintos momentos, líderes y autoridades locales han solicitado al Municipio de Guayaquil retorne la piedra a la Isla Puná, y de esa manera crear un museo de sitio, pero esto constituye parte de una lucha social que aún no ha finalizado.

La isla Puná es la tercera más grande del Ecuador después de Isabela y Santa Cruz, que están en las Galápagos. Esto constituye uno de los atractivos de la isla. Por esto sus habitantes desde hace muchos años intentan potencializar las actividades turísticas, con resultados medios. Así, en el 2010 , el Comité de Desarrollo Cacique Tumbalá impulsó la adquisición de tres lanchas rápidas para servir a la población, como parte del Proyecto de Transporte Turístico entre Guayaquil. Su objetivo es incentivar el turismo de la zona y brindar rápida transportación a los habitantes de la Isla. Hasta este momento, se han incrementado las frecuencias y número de botes para el transporte hacia la isla. Además, existe una gran flota pesquera de bajo calado, que suelen prestar sus servicios para el transporte de turistas cuando es requerido, y una frecuencia de botes diaria desde la población de Posorja hacia la comuna de Cauchiche.

Actualmente, en la cabecera parroquial, una hostería y varios restaurantes que brindan servicio a los turistas, las instalaciones son sencillas pero cómodas. También es posible encontrar propuestas para paseos por el interior 
de la isla, sus manglares y playas. Esta iniciativa de desarrollar infraestructura turística se replica en otras comunas, tal es el caso de la comuna de Cauchiche, donde en el 2013 se construyó una pequeña hostería con la finalidad de impulsar el agroturismo. En el 2017, el Ministerio de Turismo, a través de su Coordinación zonal 5, entregó el certificado de registro como Centro de Turismo Comunitario (CTC).

Otro de los atractivos, es el sector de La Concordia en la Isla Puná, sitio muy frecuentado. Allí llega una de las rutas fluviales establecidas por la Dirección Municipal de Turismo. Esta frecuencia en el transporte contribuyó en resaltar varios de los sitios de esta cabecera parroquial, Puná Nueva. En la Nueva Puná, cabecera parroquial en el 2014, se inauguró el malecón, con quioscos alrededor y baterías sanitarias, esto amplio la extensión del malecón lo cual resalta el paisaje y la integración de la naturaleza con la población.

Así también se han hecho arreglos en el ornato de la parte más alta de la población, sitio en el cual se colocó un busto del Cacique Tumbalá en octubre del 2017. Este es un personaje heroico del pueblo puneño. Este cacique se caracterizó por rechazar la colonización española a mediados del siglo XVI, y no doblegarse ante el domino español. En sí la comunidad tiene mucho bagaje cultural que brindar. Su identificación identitaria como Punáe y de descendencia étnica Huancavilca, mantiene viva tradiciones y costumbres propias de su cultura, con referentes históricos e idílicos.

A partir de esta breve descripción de los recursos existentes en la Isla Puná, es viable que surjan proyectos que incentiven las actividades turísticas y rutas de viaje a través de sus distintos ámbitos naturales y culturales. Podrían realizarse caminatas y estancias temporales entre uno y otro sitio arqueológico, intercalándolo con la experiencia naturalista al resaltar la belleza y características de cada uno de los hábitats circundantes. Y establecimiento de senderos ecológicos, a fin de conectar los diferentes atractivos naturales y arqueológicos de la isla.

Tal como ya fue expresado previamente, la isla cuenta con una considerable variedad de ecosistemas que dan forma a su patrimonio natural. El área de manglar comprende aproximadamente 14,343.96 hectáreas, los salitrales abarcan aproximadamente 871,42 hectáreas y las planicies ocupan aproximadamente una extensión aproximada de 11,382.69 hectáreas (Chancay, 2017, pp. 75-80). Con apoyo de varias instancias, el Ministerio del Ambiente a través de la Subsecretaría de Patrimonio Natural y Programa Socio Bosque, ha creado el área de bosque protector, para la captura de carbono, 
mismo que está constituido por 4533 h. Se encuentra ubicado en las comunas: Campo Alegre, Comuna Ancestral Indígena Aguas Piedras del Pueblo Punáe, Comuna Indígena Río Hondo, Comuna Indígena Bellavista de Puná y Comuna Cauchiche (PDOT, 2015). Esto nos indica que existe un reconocimiento desde las instituciones públicas sobre la diversidad ecosistémica y la necesidad de proteger el patrimonio natural presente en la Isla Puná.

Pero no todo es positivo, ya que en la isla existen muchos problemas sociales, entre los cuales resaltaremos los siguientes: ausencia de un servicio de tratamiento de aguas servidas (gran parte de la población tiene instalados pozos sépticos y un pequeño porcentaje descarga directamente hacia el mar); inseguridad ciudadana e inseguridad fluvial; aumento en el consumo de drogas, entre otros. Los pobladores argumentan que cada vez es más frecuente el uso de estupefacientes en la juventud, lo cual causa preocupación a la población isleña. Las autoridades de la zona denuncian que el área es utilizada como vía de tránsito para tráfico de drogas, lo que aumenta una percepción de inseguridad en la ciudadanía que habita la zona como para los que la visitan. Y existe la presencia de robos constantes a los pescadores de la Isla Puná, especialmente de sus motores e implementos de pesca por parte de piratas que operan en el Golfo de Guayaquil.

Por otro lado, la tala ilegal de bosque de mangle por parte de propietarios inescrupulosos de camaroneras, constituye una de las causas principales de la degradación de los ecosistemas (una de las áreas recientemente afectadas se encuentra en la Comuna Campo Alegre). Los ecosistemas de mangle son las áreas más frágiles y amenazadas, por lo que se mantiene un acuerdo entre el Ministerio del Ambiente y la asociación de pescadores artesanales de la Comuna Campo Alegre.

Ante estas situaciones, las autoridades de la parroquia se proponen una gestión efectiva, que potencie lograr el mejoramiento de los recursos, para poder ser eficientes y eficaces en la gestión de las demandas de la población (PDOT Puná, 2015, p. 34). Pero una de las dificultades que encuentran estas iniciativas es la falta de apoyo del resto del aparato gubernamental y de la empresa privada. Por ende, la cantidad de recursos que se requieren para potencializar la isla como destino turístico son pocos. La conectividad de telefonía celular e internet es insuficiente, debe extenderse y potencializarse, sumando la inclusión de procesos de formación en el manejo de redes y propuestas de promoción de los recursos de la isla Puná, no sólo por parte de las autoridades sino también por los habitantes de este territorio. Este aspecto es 
importante, ya que hasta el momento no existen canales de promoción de la lista, ni formales ni informales.

El presidente parroquial ha creado una página de la parroquia en Facebook, pero la misma no es manejada de forma constante pues el gobierno de la parroquia no cuenta con un equipo de comunicación, por ende se requiere de procesos de capacitación de la población en el uso de redes sociales y temas turísticos, además de establecerse alianzas con actores externos que permitan fortalecer la gestión turística y la promoción de los recursos de la isla, acciones que llamen la atención de los viajeros y turistas de aventura y naturalistas.

Así, el impulso de la actividad turística constituye uno de los ítems de discusión actual en la comunidad, aunque aún no se han contemplado por parte de las autoridades el tipo de impacto que tiene el desarrollo de estas actividades, impactos que abarcan desde la transformación cultural y desgaste de los recursos naturales, y la cantidad de recursos requeridos para el impulso de la difusión turística y las acciones de conservación del patrimonio que esto supone para la población.

\section{Conclusiones}

La vida de las comunidades y grupos sociales en el mundo se ha transformado bajo el paraguas de la tecnología. Esta nueva herramienta agilitó la difusión de información y los procesos comunicacionales en la sociedad. Nuevas oportunidades se vislumbran para los habitantes de la Isla Puná y sus autoridades, alrededor del aprovechamiento de sus recursos naturales y humanos en su potencialización económica a través de una actividad como el turismo, pero a la vez esto implicaría la generación de conflictos sociales e impactos ambientas al incrementarse la frecuencia y porcentaje de personas en el territorio, especialmente en referencia a transformaciones culturales y cambio en los intereses de la población.

El internet, y en específico las redes sociales, son cruciales para hacer conocer en el mundo la existencia de una oferta turística, esta es una de las oportunidades que brinda la riqueza cultural y ambiental de la isla a sus pobladores. Una oferta turística es una opción de crecimiento económico esperanzadora para sus habitantes y autoridades. Esta actividad podría generar plazas de trabajo, pero se requiere apertura en la captación de fondos y préstamos para el desarrollo de dicha actividad en la isla Puná. Así, la tecnología 
reduce la distancia entre el tiempo y el espacio, donde la comunicación digital ha terminado por ampliar nuestros mundos. La comunicación y sus usos, se convierte potencial activo que puede ser utilizado para la promoción de las bellezas y potencialidades turísticas de la Isla, a través de proyectos de difusión cultural por internet y visitas in situ.

Las acciones de protección del patrimonio natural y cultural son relevantes en el sentido de que, forman parte esencial de la supervivencia de las personas. El internet y sus usos mediáticos contribuyen a visualizar a las comunidades, a la vez impulsar actividades como el turismo. El desarrollo de esas actividades brindaría recursos no solo a la población, sino también se transformaría en un catalizador de acciones para la conservación del registro arqueológico, la presentación de expresiones culturales desde las comunidades y para la conservación de los ecosistemas de la isla. Más debe tenerse en cuenta que, existe una contradicción entre impulsar el desarrollo y el crecimiento de las actividades productivas industriales, frente al requerimiento de conservación de los recursos naturales y culturales intrínseca a la cosmovisión presente en el turismo.

De esta forma, la comunicación digital permite difundir y publicitar la riqueza de un pueblo, tanto cultural como natural, a la vez de potencializar una actividad económica como el turismo, pero debe tomarse las precauciones necesarias que reduzcan el impacto negativo que puede generar un incremento de las visitas turísticas en la isla Puna, esto implicaría un trabajo de prevención por parte de las autoridades y de la sociedad en general, labor que aún se encuentra en desarrollo por parte de los habitantes de la parroquia Puná.

\section{Bibliografía}

Aleto, T. \& Elwell, K. 1990. Informe Final de Investigación Arqueológica en los Recintos de Bellavista y Aguas Piedras, Isla Puná, Guayas. Bloomsburg University of Pennsylvania. Guayaquil: INPC Subdirección del Litoral.

Álvarez, R. (2014). Cerro Yanzún I: un sitio funerario manteño-huancavilca al norte de la isla Puná. En Estudios Multidisciplinarios en cinco espacios prehispánicos tardios del Ecuador. Quito: Senescyt-INPC-Universidad de Cuenca.

Castells, M. (2003). Internet, libertad y sociedad: una perspectiva analítica. Polis, Revista de la Universidad Bolivariana, 1(4). Recuperado de https://goo. gl/GDB7nm 
Catalano, B. \& Tottino L. (2016). Modernidad y globalización: una perspectiva a través del turismo. Unidad Sociológica, 6(2). Recuperado de https://goo. $\mathrm{gl} / \mathrm{xd} 2 \mathrm{Xr} 9$

Chancay-Vásquez, J. (2017). Sitios arqueológicos identificados en la Isla Puná: Relación entre asentamientos humanos y ecología (Tesis de Maestría). Escuela Superior Politécnica del Litoral. Facultad de Ingeniería en Ciencias de la Tierra. Guayaquil, Ecuador.

Cioce, C. \& Silva. Y. (2015). Turismo como fenómeno humano. Principios para pensar en la ecosocioeconomía. Turydes. Revista turismo y desarrollo local, 8(19). Recuperado de https://goo.gl/mpvqAS

Crespo, V. (2014). Ecosistemas y manejo de recursos en la isla Puná. En: Estudios Multidisciplinarios en cinco espacios prehispánicos tardios del Ecuador. Quito. Senescyt-INPC-Universidad de Cuenca.

Delgado, J. (2014) La isla Puná o Santiago en el período de Integración: 1400-1531. En: Estudios Multidisciplinarios en cinco espacios prehispánicos tardíos del Ecuador. Quito. Senescyt-INPC-Universidad de Cuenca.

Deux, M. \& Vannini. P. (2016). Manual de tecnologías abiertas para la gestión de organizaciones de la economía social y solidaria. Buenos Aires: Editorial de la Universidad Nacional de General Sarmiento.

Díaz, P. \& López, B. (2012). La promoción turística oficial en Internet y su relación con el desarrollo turístico de los destinos: Una aplicación a las Ciudades medias de Andalucía. Revista de Estudios Regionales, 93. Recuperado de https://goo.gl/6xCXor

Harris, M. (2007 [1989]). Teorías sobre la cultura en la era postmoderna. Barcelona. Crítica.

Kulesz, O. (2017). La cultura en el entorno digital. Evaluar el impacto en América Latina y España. París: UNESCO.

Labate, C. \& Arrueta, C. (2017). Comunicación digital: redes sociales, nuevas audiencias y convergencia: desafios y oportunidades para la industria, el Estado y los usuarios. San Salvador de Jujuy: Editorial de la Universidad Nacional de Jujuy-EDIUNJU.

Martín-Barbero, J. (04/2015). ¿Desde dónde pensamos la comunicación hoy? Chasqui. Revista Latinoamericana de Comunicación, 128, 13-29. Recuperado de https://goo.gl/VVBJg4

Martínez, B. (2006). Homo Digitalis. Etnografía de la Cibercultura. Universidad de los Andes. Bogotá: Ediciones Uniandes. 
Moreno, C. (2015). Co Crear con el digital en un mundo en mutación. Communication Papers. Media Literacy \& Genders Studies. CP, 4(8) (Monográfico II). Recuperado de https://goo.gl/jh9SMN

Plaza-Vanegas, G. (2017). Matriz de Problemáticas y Propuestas respecto a la situación de la Parroquia Isla Puná en el 2017. Ecuador: Consejo Nacional de Gobiernos Parroquiales Rurales del Ecuador (CONAGOPARE). Dirección de Planificación y Cooperación.

PDOT Puná (2015). Plan de Desarrollo y Ordenamiento Territorial de la Parroquia Rural de Puná 2015-2019.

Quiñones-Bonilla, F. (12/2005). De la cultura a la cibercultura. Revista Hallazgos, 4, 174-190. Recuperado de https://goo.gl/XK3Zxd

Requena, K. \& Muñoz, J. (2006). El turismo e internet, factores de desarrollo en países subdesarrollados. Actualidad Contable FACES, 9(12). Recuperado de https://goo.gl/axXYJS

Sánchez, F. (2013). Informe Final del Proyecto "Excavación de Los Sitios Arqueológicos NVE1-001 Y MVF3-01, Parroquia Puná. Instituto Nacional de Patrimonio Cultural-INPC, Guayaquil.

Sandoval, E. (2007). Cibersocioantropología. Revista Argentina de Sociología, 5(9), 64-89. Recuperado de https://goo.gl/BCLdVc

Torres, E. (03/2014). Comunicación y cultura en Manuel Castells: exploraciones del periodo 1996-2009. Athenea Digital, 14(1), 355-373. Recuperado de https://goo.gl/m48g54

Zabala, D., Nato, R. \& Rosero, P. (2014) Caracterización geográfica de la isla Puná. En: Estudios Multidisciplinarios en cinco espacios prehispánicos tardíos del Ecuador. Quito. Senescyt-INPC-Universidad de Cuenca.

Fecha de recepción: 2018/11/20; Fecha de aceptación: 2019/02/18;

Fecha de publicación: 2019/03/01 